\title{
MONOTONICITY, CONVEXITY AND BOUNDS INVOLVING THE BETA AND RAMANUJAN $R$-FUNCTIONS
}

\section{Ti-Ren Huang, Lu Chen, Shen-Yang TAn And Yu-Ming Chu*}

\begin{abstract}
In the article, we provide several new asymptotical sharp bounds for the functions involving the Beta function and Ramanujan $R$-functions via the monotonicity and convexity properties of certain combinations defined in terms of polynomials, Beta and Ramanujan $R$ functions.
\end{abstract}

Mathematics subject classification (2020): 33C05, 26D20.

Keywords and phrases: Ramanujan $R$-function, beta function, monotonicity, convexity.

\section{REFERENCES}

[1] M. Abramowitz, I. A. Stegun, Handbook of Mathematical Functions with Formulas, Granphs and Mathematical Tables, Dover, New York, 1965.

[2] G. D. Anderson, M. K. Vamanamurthy, M. Vuorinen, Conformal Invariants, Inequalities, and Quasiconformal Mappings, John Wiley and Sons, New York, 1997.

[3] G. D. Anderson, S.-L. QIU, M. K. VAMANAmurthy, Elliptic integral inequalities, with applications, Constr Approx. 1998, 14 (2), 195-207.

[4] G. D. Anderson, R. W. Barnard, K. C. Richards, M. K. Vamanamurthy, M. Vuorinen, Inequalities for zero-balanced hypergeometric functions, Trans. Amer. Math. Soc. 1995, 347, 17131723.

[5] G. D. Anderson, S.-L. Qiu, M. K. Vamanamurthy, M. Vuorinen, Generalized elliptic integrals and modular equations, Pacific J. Math. 2000, 192 (1), 1-37.

[6] G. E. Andrews, R. Askey, R. Roy, Special Functions, Cambridge Univiversity Press, Cambridge, 1999.

[7] J. M. Borwein, P. B. Borwein, Pi and the AGM, John Wiley \& Sons, New York, 1987.

[8] B. C. BERndT, Ramanujan's Notebooks, Part II, Springer-Verlag, New York, 1989.

[9] B. C. Berndt, Ramanujan's Notebooks, Part IV, Springer-Verlag, New York, 1994.

[10] R. Balasubramanian, S. Ponnusamy, M. Vuorinen, Functional inequalities for quotients of hypergeometric functions, J. Math. Anal. Appl. 1998, 218, 256-268.

[11] W. A. DAY, On monotonicity of the relaxation functions of viscoelastic materials, Proc. Cambridge Philos. Soc. 1970, 67, 503-508.

[12] W. FelLeR, An Introduction to Probability Theory and Its Applications, Vol. II, John Wiley \& Sons, New York, 1966.

[13] T.-R. HuAng, S.-L. QIU, X.-Y. MA, Monotonicity properties and inequalities for the generalized elliptic integral of the first kind, J. Math. Anal. Appl. 2019, 469 (1), 95-116.

[14] Y.-X. Li, M. A. Ali, H. Budak, M. AbBas, Y.-M. ChU, A new generalization of some quantum integral inequalities for quantum differentiable convex functions, Adv. Difference Equ. 2021, 2021, Article 225, 15 pages.

[15] Y.-X. Li, M. H. Alshbool, Y.-P. Lv, I. Khan, M. RiZa Khan, A. IssaKhov, Heat and mass transfer in MHD Williamson nano uid ow over an exponentially porous stretching surface, Case Stud. Therm. Eng. 2021, 26, Article ID 100975, 10 pages.

[16] Y.-X. Li, A. Rauf, M. Naeem, M. A. Binyamin, A. Aslam, Valency-based topological properties of linear hexagonal chain and hammer-like benzenoid, Complexity, 2021, 2021, Article ID 9939469,16 pages. 
[17] S.-L. QIU, B.-P. Feng, Some properties of the Ramanujan constant, J. Hangzhou Dianzi Univ. 2007, 27 (3), 88-91.

[18] S.-L. QIU, X.-Y. MA, T.-R. HuAnG, Some properities of the difference between the Ramanujan constant and Beta function, J. Math. Anal. Appl., 2017, 446, 114-129.

[19] S.-L. Qiu, M.-K. Vamanamurthy, Elliptic integrals and the modulus of Grötzsch ring, Panamer. Math. J. 1995, 5, 41-60.

[20] M.-K. WANG, Y.-M. ChU, S.-L. QIU, Sharp bounds for generalized elliptic integrals of the first kind, J. Math. Anal. Appl. 2015, 429, 744-757.

[21] E. T. Whittaker, G. N. Waston, A course of Modern Analysis, Cambridge University Press, London, 1958.

[22] J. Wimp, Sequence Transformations and Their Applications, Academic Press, New York, 1981.

[23] P.-G. Zhou, S.-L. QIU, G.-Y. TU, Y.-L. Li, Some properties of the Ramanujan constant, J. Zhejiang Sci-Tech Univ. 2010, 27 (5), 835-841. 